Research Paper

\title{
Effect of lymph nodes count in node-positive gastric cancer
}

\author{
Wenjie Zhang1,2,3* Guangyan Zhangyuan ${ }^{1,3^{*}}$, Jincheng Wang1,2,3* Kangpeng Jin ${ }^{1}$, Yang Liu ${ }^{1,2}$, Fei Wang1,2, \\ Weiwei $\mathrm{Yu}^{1,2}$, Haitian Zhang ${ }^{1,2}$, Guoqiang $\mathrm{Li}^{1}$, Decai $\mathrm{Yu}^{1}$, Huihui Chen ${ }^{1}$, Qingxiang $\mathrm{Xu}^{1,2}{ }^{\circledR}$, Beicheng \\ Sun $1,2,3$ 四 \\ 1. Department of Hepatobiliary Surgery, The Affiliated Drum Tower Hospital of Nanjing University Medical School, Nanjing 210029, Jiangsu Province, P.R. \\ China \\ 2. Department of Hepatobiliary Surgery of Drum Tower Clinical Medical College, Nanjing Medical University, Nanjing, China. \\ 3. Liver Transplantation Center, The First Affiliated Hospital of Nanjing Medical University, Nanjing, Jiangsu Province, P.R. China. \\ *The authors contributed equally to this work \\ $\square$ Corresponding authors: sunbc@nju.edu.cn (B.S.).xqx2016nj@sina.com (Q.X). Telephone: 86-25-68136746; Fax: 86-25-86560946
}

(c) The author(s). This is an open access article distributed under the terms of the Creative Commons Attribution License (https://creativecommons.org/licenses/by/4.0/). See http://ivyspring.com/terms for full terms and conditions.

Received: 2018.10.26; Accepted: 2019.08.15; Published: 2019.09.07

\begin{abstract}
Background: The retrieved lymph node (LN) count has been confirmed as a prognostic indicator in various cancers. However, the correlation between LN counts and patient prognosis in gastric cancer with node-positive is not fully studied.

Methods: A total of 8475 patients undergoing gastrectomy in Surveillance, Epidemiology, and End Results Program (SEER)-registered gastric cancer were analyzed. Kaplan-Meier methods and multivariable Cox regression models were used to analyze long-term outcomes and risk factors. Moreover, nomograms including LN counts were established to predict overall survival (OS) and cancer-specific survival (CSS), and Harrell's concordance index (c-index) was adopted to evaluate prediction accuracy.

Results: Patients were stratified into 1-6, 7-14, and > 14 subgroups according to the optimal cutoff for retrieved LNs in terms of 5-year CSS. Further analysis indicated that higher LN counts were an independent predictor of longer survival in each $\mathrm{N}$ category. Nomograms on CSS and OS were established according to all significant factors, and c-indexes were 0.663 and $0.654(\mathrm{P}<0.001)$, respectively.

Conclusions: These results indicated that the more the LNs retrieved, the better the survival would be. Nomograms incorporating $\mathrm{LN}$ counts can be recommended as practical models to provide more accurate prognostic information for GC patients.
\end{abstract}

Key words: nomograms; lymph nodes; gastric cancer

\section{Introduction}

Gastric cancer (GC) ranks fourth in frequency in the world and is globally the second leading cause of cancer-related death [1, 2]. GC is the most common malignancy in Latin America and Asia, and its incidence is nearly 10-fold higher than in the US [3]. According to the 7th edition of the AJCC TNM classification, the minimum number of retrieved LNs is not defined [4]. Meanwhile, the number of metastatic LNs was validated as an independent prognostic factor after surgical resection $[5,6]$. However, whether more retrieved LNs can be linked to accurate staging is controversial. In addition, there is doubt regarding the recommended minimum retrieval of 15 LNs for GC [7]. Some studies sought to investigate the optimal LNs retrieval cutoff in node-negative GC, but few studies have focused on node-positive patients in a large population [8].

The objective of this retrospective study was to assess the effect of retrieved LN counts on the long-term survival outcome in node-positive gastric patients, and to explore the optimal retrieved LNs cutoff value. In this study, we searched the Surveillance, Epidemiology, and End Results (SEER) population-based database and analyzed the 
clinicopathological characteristics and cancer-specific survival of these subgroups. We also used the X-tile program to determine the optimal cutoff.

\section{Methods}

\section{Patient selection}

Data were obtained from the Surveillance Epidemiology and End Results (SEER) Program of the United States National Cancer Institute. The current SEER database consists of 18 population-based cancer registries that represent approximately $26 \%$ of the population in the United States. SEER data contain no identifiers and are publicly available for studies of cancer-based epidemiology and survival analysis.

Inclusion criteria included the following: (1) patients were diagnosed from 2004 to 2012; (2) the site code was limited to stomach; (3) underwent surgical resection; (4) age > 18 years old; (5) histology code was limited to adenocarcinoma (8140/3, 8144/3, $8255 / 3,8211 / 3,8260 / 3,8263 / 3)$, mucinous adenocarcinoma $(8480 / 3)$, and signet ring cell carcinoma (8490/3); (6) at least with one LN retrieval; (7) information on CSS and OS available. The primary endpoint of the study is 5-year CSS, which was calculated from the date of diagnosis to the date of cancer-specific death. Cancer-specific deaths were treated as events, and deaths from other causes were treated as censored observations. The median followup of patients was calculated from the date of diagnosis to the date of cancer-specific death.

This study was based on public data from the SEER database; we obtained permission to access research data files with the reference number 10504-Nov2014. The data did not include the use of human subjects or personal identifying information. Thus, no informed consent was required for this part of the study. The methods were carried out in accordance with the approved guidelines in this study. Ethical approval was obtained from the institutional review board of Nanjing Medical University.

\section{Identification of the optimal cutoff point of retrieved LNs}

The retrieved LNs cutoff points were produced and analyzed using the X-tile program, which identified the cutoff with the minimum $p$ values from log-rank $\chi^{2}$ statistics for the categorical LN counts in terms of survival.

\section{Statistical analysis}

Categorical variables were summarized using frequency (\%). A comparison of the categorical variables between LNs count subgroups was conducted using Pearson's $\chi^{2}$ test. Continuous variables were compared using the Mann-Whitney $U$ test. Survival curves were generated using the Kaplan-Meier method; differences between the curves were analyzed by the log-rank test. Multivariable Cox proportional hazards regression models were used to assess potential risk factors for CSS. Cox stepwise regression analysis was also performed to determine predictive factors for gastric cancer prognosis, with a significance level of 0.05 for entering and 0.10 for removing the respective explanatory variables. Nomograms for possible prognostic factors associated with CSS and OS were established by $R$ software, and the model performance for predicting outcome was evaluated by Harrell's concordance index (c-index), which is a measure of discrimination.

All statistical analyses were performed using the statistical software package SPSS for Windows, version 17 (SPSS Inc., Chicago, IL, USA). The results were considered statistically significant when a two-tailed test provided a P-value of less than 0.05.

\section{Results}

\section{Patient Characteristics}

We identified 8475 eligible patients with GC meeting the eligibility criteria in the SEER database, including 5404 male and 3071 female. All patients had at least one LN examined. There were 2738 patients with N1 stage, 2493 patients with N2 stage, 2252 patients with N3a stage, and 992 patients with N3b stage. Patient demographics and pathological features are summarized in Table 1. Patients had a higher rate of poor/ anaplastic grade tumors, a higher ratio of cardia and gastric antrum tumors, a higher proportion of adenocarcinoma and T3/4 tumor stage across all the $\mathrm{N}(+)$ patients $(\mathrm{P}<0.001)$. The median number of LNs examined was 17.89 (range, 9-23). The median positive LN count was 7.19 (range, 2-10).

\section{Identification of minimum number of retrieved LNs in node-positive patients}

X-tile plots were constructed and the maximum of chi-square log-rank values of $154.244(\mathrm{P}<0.001)$ was achieved when applying 6 and 14 as the cutoff value of retrieved LNs. This value can be used to divide the cohort into high, middle and low risk subsets in terms of gastric cancer-specific survival (GCSS), which were 20.3\%, 29.0\% and 32.6\%, respectively $(\mathrm{P}<0.001)$ (Fig. 1). Then, to investigate the impact of different LN counts on GCSS, we treated the number of $\mathrm{LN}$ counts as a continuous variable and analyzed the number of retrieved LNs from 2 to 20 . The number of retrieved LNs was an independent prognosis factor for GC, and patients with 15 or more 
LNs retrieved had a relative $14.4 \%$ improvement in 5-year GCSS compared to those with 6 less LNs retrieved (32.6\% versus $18.2 \%$ ). The 5 -year GCSS of patients with $\mathrm{N}$ or more nodes increased gradually when $\mathrm{N}$ reaching 14 . After the number 15 , the survival rates were roughly stable between the compared groups (Table 2).

\section{Effect of LN counts on GCSS rates in the SEER database}

The univariate log-rank test showed that, beside of the number of retrieved LNs, other clinicopathological factors, including age more than 60 years, White race, poor/undifferentiated tumor grade, overlapping lesion of stomach, mucinous and signet-ring cancer as well as advanced TN stages were regarded as significant risk factors for 5-year CSS rate $(\mathrm{P}<0.001)$. Multivariate analysis with Cox regression demonstrated that more retrieved LNs exhibited survival advantage (LNs: 7-14, hazard ratio (HR) 0.586; 95\% confidence interval [CI] 0.536-0.640; LNs: $\geq 15$, HR 0.390; 95\% CI 0.356-0.427) $(\mathrm{P}<0.001)$ (Table 3).

\section{Prognostic nomogram for CSS and OS}

To predict CSS and OS in GC patients, the external validation of nomograms was performed and predictive factors were determined by cox stepwise regression analysis (Fig. 3A and 3B) [9]. Each variable was assigned a score at the top of scale. By counting the total score, we were able to draw a straight line down to predict 3-year and 5-year probability of survival for a patient at each time point. The Harrell's c-indexes to predict CSS and OS prediction were 0.663 (95\% CI: 0.655-0.671) and 0.654 (95\% CI: 0.646-0.662) $(\mathrm{P}<0.001)$, which were significantly higher than those of the model without the variable of dissected LNs (CSS: 0.663 versus 0.64 ; OS: 0.654 versus 0.63$)(\mathrm{P}<$ 0.001). Calibration curves for two nomograms (Fig. $3 \mathrm{C}$ and $3 \mathrm{D}$ ) revealed no deviations from the reference line and no need of recalibration. The decision curve analysis indicated that for most of the threshold probabilities for 5-year CSS and OS, with LN count nomogram achieved a greater net benefit compared with without LN count (Fig. S1).

Table 1. Demographic and tumor characteristics of patients with node positive gastric cancer

\begin{tabular}{|c|c|c|c|c|c|c|c|c|c|c|c|}
\hline \multicolumn{11}{|c|}{ Subgroup } & \multirow{4}{*}{ P value } \\
\hline & \multirow{2}{*}{\multicolumn{2}{|c|}{$\begin{array}{l}\text { All Patients } \\
\mathrm{n}=8475\end{array}$}} & \multirow{2}{*}{\multicolumn{2}{|c|}{$\begin{array}{l}\mathrm{N} 1 \\
\mathrm{n}=2738\end{array}$}} & \multirow{2}{*}{\multicolumn{2}{|c|}{$\begin{array}{l}\mathrm{N} 2 \\
\mathrm{n}=2493\end{array}$}} & \multirow{2}{*}{\multicolumn{2}{|c|}{$\begin{array}{l}\mathrm{N} 3 \mathrm{a} \\
\mathrm{n}=2252\end{array}$}} & \multicolumn{2}{|l|}{$\mathrm{N} 3 \mathrm{~b}$} & \\
\hline & & & & & & & & & \multicolumn{2}{|c|}{$\mathrm{n}=992$} & \\
\hline Characteristic & No. & $\%$ & No. & $\%$ & No. & $\%$ & No. & $\%$ & No. & $\%$ & \\
\hline \multicolumn{3}{|l|}{ Sex } & & & & & & & & & 0.016 \\
\hline male & 5404 & 63.8 & 1775 & 64.8 & 1613 & 64.7 & 1426 & 63.3 & 590 & 59.5 & \\
\hline female & 3071 & 36.2 & 963 & 35.2 & 880 & 35.3 & 826 & 36.7 & 402 & 40.5 & \\
\hline \multicolumn{3}{|l|}{ Age } & & & & & & & & & $<0.001$ \\
\hline$<60$ & 2924 & 34.5 & 846 & 30.9 & 843 & 33.8 & 876 & 38.9 & 359 & 36.2 & \\
\hline$\geq 60$ & 5551 & 65.5 & 1892 & 69.1 & 1650 & 66.2 & 1376 & 61.1 & 633 & 63.8 & \\
\hline \multicolumn{3}{|l|}{ Race } & & & & & & & & & $<0.001$ \\
\hline White & 5629 & 66.4 & 1856 & 67.8 & 1681 & 67.4 & 1453 & 64.5 & 639 & 64.4 & \\
\hline African American & 1170 & 13.8 & 381 & 13.9 & 364 & 14.6 & 301 & 13.4 & 124 & 12.5 & \\
\hline Others & 1676 & 19.8 & 501 & 18.3 & 448 & 18.0 & 498 & 22.1 & 229 & 23.1 & \\
\hline \multicolumn{3}{|l|}{ Location } & & & & & & & & & $<0.001$ \\
\hline Cardia, NOS & 2198 & 15.9 & 864 & 31.6 & 711 & 28.5 & 474 & 21.0 & 149 & 15.0 & \\
\hline Fundus of stomach & 255 & 3.0 & 76 & 2.8 & 92 & 3.7 & 56 & 2.5 & 31 & 3.1 & \\
\hline Body of stomach & 661 & 8.0 & 202 & 7.4 & 185 & 7.4 & 180 & 8.0 & 94 & 9.5 & \\
\hline Gastric antrum & 2118 & 25.0 & 652 & 23.8 & 631 & 25.3 & 596 & 26.5 & 239 & 24.1 & \\
\hline Pylorus & 403 & 4.8 & 130 & 4.7 & 129 & 5.2 & 117 & 5.2 & 27 & 2.7 & \\
\hline Lesser curvature of stomach NOS & 952 & 11.2 & 295 & 10.8 & 248 & 9.9 & 286 & 12.7 & 123 & 12.4 & \\
\hline Greater curvature of stomach NOS & 402 & 4.7 & 128 & 4.7 & 108 & 4.3 & 111 & 4.9 & 55 & 5.5 & \\
\hline Overlapping lesion of stomach & 756 & 8.9 & 174 & 6.4 & 182 & 7.3 & 245 & 10.9 & 155 & 15.6 & \\
\hline Stomach, NOS & 730 & 8.6 & 217 & 7.9 & 207 & 8.3 & 187 & 8.3 & 119 & 12.0 & \\
\hline \multicolumn{3}{|l|}{ Pathological grading } & & & & & & & & & $<0.001$ \\
\hline High/ Moderate & 1985 & 23.4 & 853 & 31.2 & 611 & 24.5 & 404 & 17.9 & 117 & 11.8 & \\
\hline Poor/ Anaplastic & 6171 & 72.8 & 1731 & 63.2 & 1813 & 72.7 & 1782 & 79.1 & 845 & 85.2 & \\
\hline Unknown & 319 & 3.8 & 154 & 5.6 & 69 & 2.8 & 66 & 2.9 & 30 & 3.0 & \\
\hline \multicolumn{3}{|l|}{ Histotype } & & & & & & & & & $<0.001$ \\
\hline Adenocarcinoma & 5962 & 70.3 & 2110 & 77.1 & 1825 & 73.2 & 1474 & 65.5 & 553 & 55.7 & \\
\hline Mucinous / Signet ring cell & 2513 & 29.7 & 628 & 22.9 & 668 & 26.8 & 778 & 34.5 & 439 & 44.3 & \\
\hline \multicolumn{3}{|l|}{ pT stage } & & & & & & & & & $<0.001$ \\
\hline $\mathrm{T} 1$ & 581 & 6.9 & 373 & 13.6 & 159 & 6.4 & 41 & 1.8 & 8 & 0.8 & \\
\hline $\mathrm{T} 2$ & 786 & 9.3 & 412 & 15.0 & 227 & 9.1 & 122 & 5.4 & 25 & 2.5 & \\
\hline $\mathrm{T} 3$ & 3596 & 42.4 & 1090 & 39.8 & 1169 & 46.9 & 1007 & 44.7 & 330 & 33.3 & \\
\hline $\mathrm{T} 4$ & 3460 & 40.8 & 820 & 29.9 & 934 & 37.5 & 1078 & 47.9 & 628 & 63.3 & \\
\hline $\mathrm{Tx}$ & 52 & 0.6 & 43 & 1.6 & 4 & 0.2 & 4 & 0.2 & 1 & 0.1 & \\
\hline No. of LNs dissected & \multicolumn{2}{|c|}{$17.89(9-23)$} & \multicolumn{2}{|c|}{$13.18(5-18)$} & \multicolumn{2}{|c|}{$15.91(8-20)$} & \multicolumn{2}{|c|}{$19.67(13-24)$} & \multicolumn{2}{|c|}{$31.87(22-38)$} & $<0.001$ \\
\hline No. of positive LNs & \multicolumn{2}{|c|}{$7.19(2-10)$} & \multicolumn{2}{|c|}{$1.40(1-2)$} & \multicolumn{2}{|c|}{$4.26(3-5)$} & 10.14 & & 23.80 & 27) & $<0.001$ \\
\hline
\end{tabular}



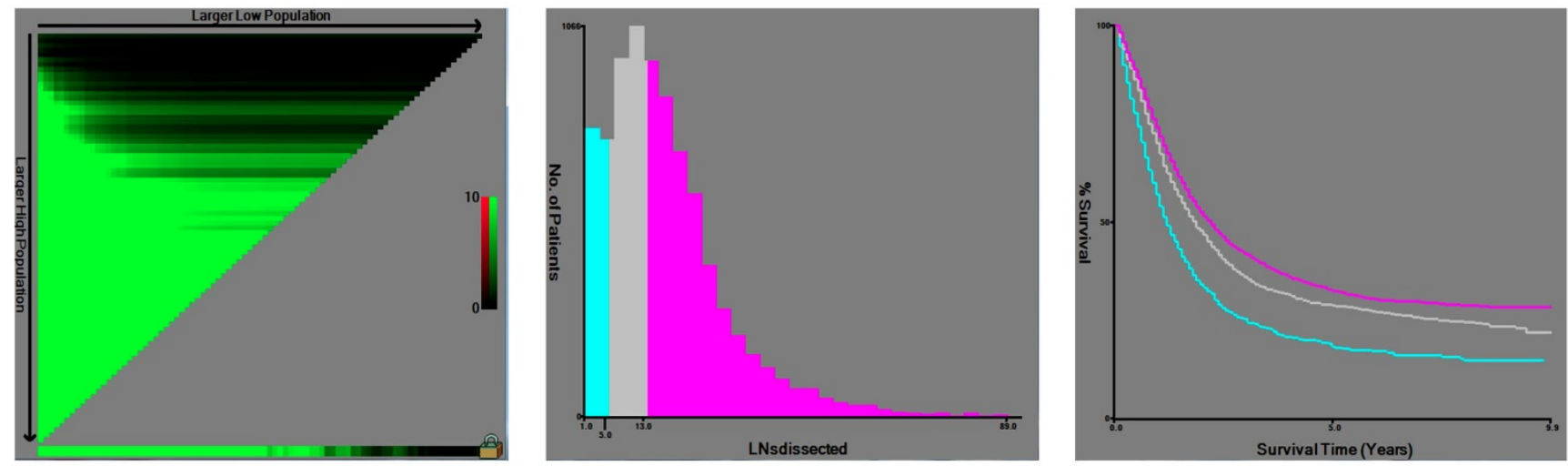

Figure 1. X-tile analysis of survival data from the SEER registry. X-tile analysis was done on patient data from the SEER registry, equally divided into training and validation sets. The optimal cut-point highlighted by the black circle in the left panels (A) is shown on a histogram of the entire cohort (middle panels) (B), and a Kaplan-Meier plot (right panels) (C). P values were determined by using the cut-point defined in the training set and applying it to the validation set. Figure 1 shows the optimal cutoff point for the lymph node positive patients (number 6 and $14, X 2=154.244, P<0.001$ )

A

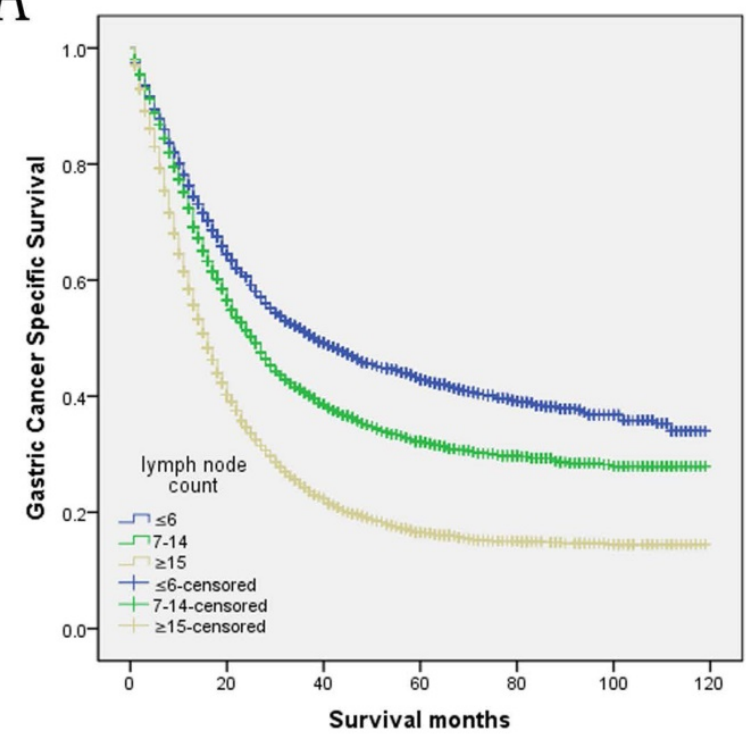

C

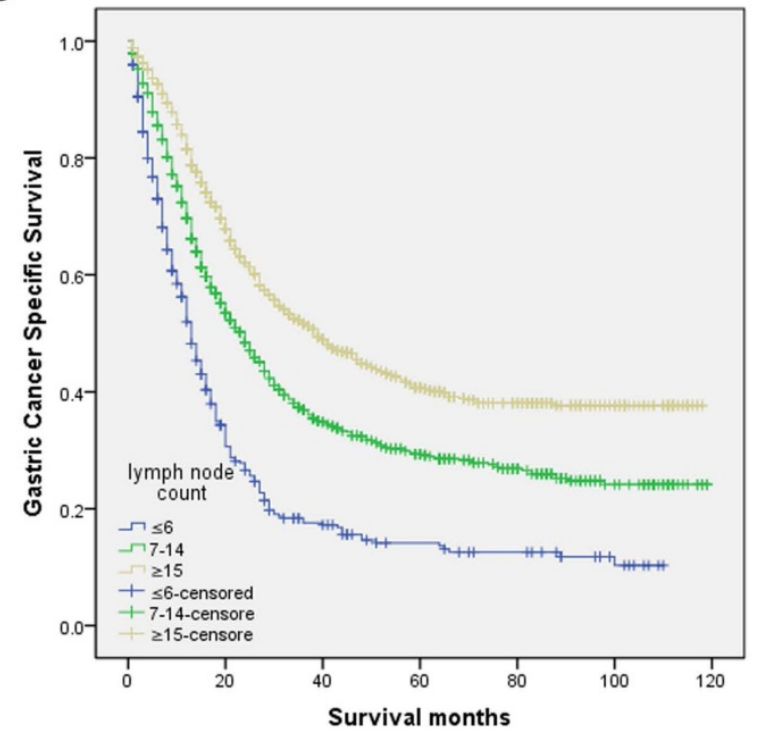

B

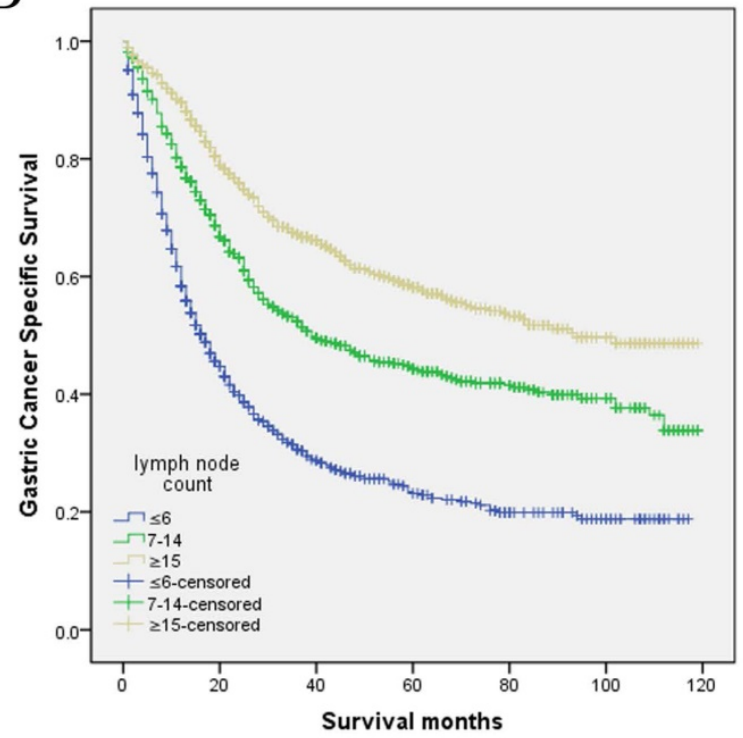

D

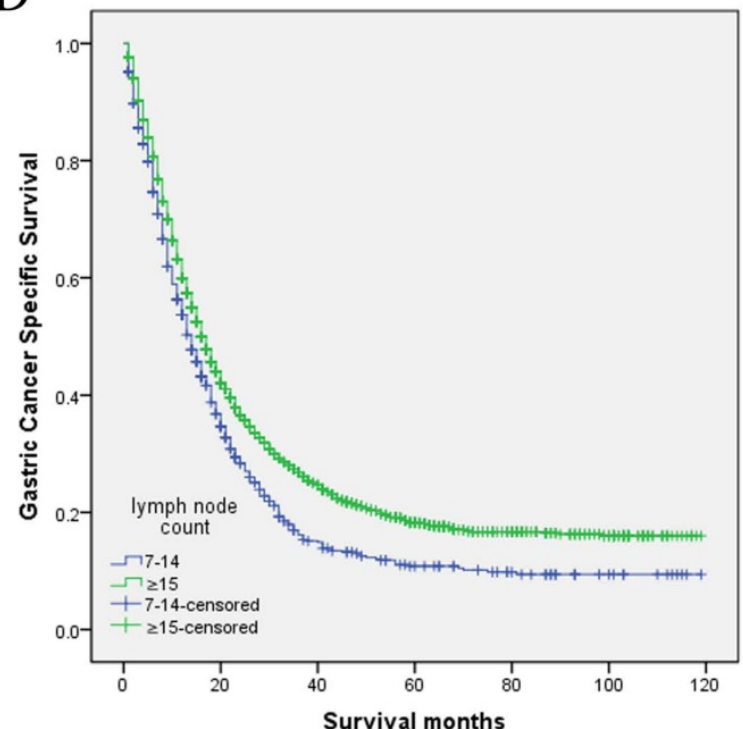

Figure 2. Log-rank tests of cause specific comparing those who had $\geq 15,7-14$, and $\leq 6$ positive lymph nodes for $A$. all stage: $X 2=491.935, P<0.001$; $B$. N1 stage: $X 2=305.678, P<0.001 ; C . N 2$ stage: $X 2=200.635, P<0.001 ; D . N 3$ stage: $X 2=29.113, P<0.001$. 
Table 2. Univariate analysis for the influence of different cutoffs on GCSS in gastric cancer.

\begin{tabular}{|c|c|c|c|c|}
\hline Cutoff & No. & 5-year GCCS & Log-rank $\chi^{2}$ & $P$ value \\
\hline$<2$ & 268 & $10.2 \%$ & 125.990 & $<0.001$ \\
\hline$\geq 2$ & 8207 & $30.3 \%$ & & \\
\hline$<3$ & 413 & $13.3 \%$ & 114.568 & $<0.001$ \\
\hline$\geq 3$ & 8062 & $30.5 \%$ & & \\
\hline$<4$ & 591 & $15.2 \%$ & 130.281 & $<0.001$ \\
\hline$\geq 4$ & 7884 & $30.7 \%$ & & \\
\hline$<5$ & 787 & $18.7 \%$ & 99.070 & $<0.001$ \\
\hline$\geq 5$ & 7688 & $30.8 \%$ & & \\
\hline$<6$ & 998 & $18.2 \%$ & 132.053 & $<0.001$ \\
\hline$\geq 6$ & 7477 & $31.2 \%$ & & \\
\hline$<7$ & 1252 & $20.3 \%$ & 118.442 & $<0.001$ \\
\hline$\geq 7$ & 7223 & $31.3 \%$ & & \\
\hline$<8$ & 1544 & $21.7 \%$ & 114.520 & $<0.001$ \\
\hline$\geq 8$ & 6931 & $31.4 \%$ & & \\
\hline$<9$ & 1808 & $22.1 \%$ & 121.692 & $<0.001$ \\
\hline$\geq 9$ & 6667 & $31.7 \%$ & & \\
\hline$<10$ & 2165 & $23.0 \%$ & 117.830 & $<0.001$ \\
\hline$\geq 10$ & 6310 & $31.9 \%$ & & \\
\hline$<11$ & 2521 & $24.7 \%$ & 83.525 & $<0.001$ \\
\hline$\geq 11$ & 5954 & $31.7 \%$ & & \\
\hline$<12$ & 2883 & $24.8 \%$ & 88.381 & $<0.001$ \\
\hline$\geq 12$ & 5592 & $32.2 \%$ & & \\
\hline$<13$ & 3234 & $25.6 \%$ & 77.568 & $<0.001$ \\
\hline$\geq 13$ & 5241 & $32.2 \%$ & & \\
\hline$<14$ & 3587 & $25.8 \%$ & 77.682 & $<0.001$ \\
\hline$\geq 14$ & 4888 & $32.5 \%$ & & \\
\hline$<15$ & 3882 & $26.2 \%$ & 70.925 & $<0.001$ \\
\hline$\geq 15$ & 4593 & $32.6 \%$ & & \\
\hline$<16$ & 4239 & $26.6 \%$ & 60.232 & $<0.001$ \\
\hline$\geq 16$ & 4236 & $32.7 \%$ & & \\
\hline$<17$ & 4557 & $26.9 \%$ & 53.464 & $<0.001$ \\
\hline$\geq 17$ & 3918 & $32.9 \%$ & & \\
\hline$<18$ & 4873 & $26.8 \%$ & 59.287 & $<0.001$ \\
\hline$\geq 18$ & 3602 & $33.5 \%$ & & \\
\hline$<19$ & 5178 & $27.7 \%$ & 42.607 & $<0.001$ \\
\hline$\geq 19$ & 3297 & $32.8 \%$ & & \\
\hline$<20$ & 5431 & $28.0 \%$ & 38.271 & $<0.001$ \\
\hline$\geq 20$ & 3044 & $32.7 \%$ & & \\
\hline
\end{tabular}

\section{Subgroup analysis of retrieved LNs effect on GCSS according to $\mathrm{pN}$ categories}

We then further analyzed the effect of retrieved LNs on GCSS rates in each stage. After stratifying by the confounding factors, the univariate analysis of retrieved LNs effect on GCSS rates showed that the retrieved LNs exhibited increased 5-year GCSS rates across several $\mathrm{N}$ subgroups $(\mathrm{P}<0.001)$. Comparing with the patients who had $\leq 6$ retrieved LNs, there was a $35.0 \%$ and $27.1 \%$ improvement in 5-year GCSS in those $\geq 15$ retrieved LNs patients in N1 and N2 stage, and stills a $7.5 \%$ improvement when compared with 7-14 retrieved LNs patients in N3 stage $(\mathrm{P}<0.001)$. Besides, the retrieved LNs were also validated as an independent predictor of survival in multivariate Cox regression in $\mathrm{N} 1$ stage (LNs $\geq 15, \mathrm{HR} 0.373,95 \% \mathrm{CI}$ $0.325-0.427, \mathrm{P}<0.001), \mathrm{N} 2$ stage (LNs $\geq 15, \mathrm{HR} 0.406$, $95 \%$ CI $0.352-0.469, \mathrm{P}<0.001)$ and N3 stage $(\mathrm{LNs} \geq 15$, HR 0.789, 95\% CI 0.719-0.865, P< 0.001) (Table 4).
Table 3. Univariate and multivariate survival analyses for evaluating the influence of the number of retrieved LNs influencing GCSS in node positive gastric cancer patients.

\begin{tabular}{|c|c|c|c|c|c|}
\hline & & $\begin{array}{l}\text { Univariat } \\
\text { analysis }\end{array}$ & & Multivariate anal & ysis \\
\hline Variable & $\begin{array}{l}\text { 5-year } \\
\text { GCCS }\end{array}$ & $\begin{array}{l}\text { Log rank } \\
\chi^{2} \text { test }\end{array}$ & $\mathbf{P}$ & $\operatorname{HR}(95 \% \mathrm{CI})$ & $\mathbf{P}$ \\
\hline Sex & & 3.059 & 0.08 & & \\
\hline Male & $30.4 \%$ & & & & \\
\hline Female & $28.5 \%$ & & & & \\
\hline Age & & 23.923 & $<0.001$ & & $<0.001$ \\
\hline$<60$ & $32.0 \%$ & & & Reference & \\
\hline$\geq 60$ & $28.4 \%$ & & & $1.295(1.221-1.373)$ & \\
\hline Race & & 51.463 & $<0.001$ & & $<0.001$ \\
\hline White & $27.6 \%$ & & & Reference & \\
\hline African American & $28.2 \%$ & & & $1.042(0.960-1.131)$ & \\
\hline Others & $37.5 \%$ & & & $0.809(0.751-0.871)$ & \\
\hline Location & & 72.114 & $<0.001$ & & 0.0251 \\
\hline Cardia, NOS & $26.1 \%$ & & & Reference & \\
\hline Fundus of stomach & $31.4 \%$ & & & $0.827(0.700-0.978)$ & \\
\hline Body of stomach & $30.5 \%$ & & & $0.769(0.687-0.861)$ & \\
\hline Gastric antrum & $32.6 \%$ & & & $0.778(0.718-0.842)$ & \\
\hline Pylorus & $29.7 \%$ & & & $0.857(0.748-0.982)$ & \\
\hline $\begin{array}{l}\text { Lesser curvature of } \\
\text { stomach NOS }\end{array}$ & $37.1 \%$ & & & $0.693(0.625-0.768)$ & \\
\hline $\begin{array}{l}\text { Greater curvature of } \\
\text { stomach NOS }\end{array}$ & $31.2 \%$ & & & $0.812(0.707-0.931)$ & \\
\hline $\begin{array}{l}\text { Overlapping lesion of } \\
\text { stomach }\end{array}$ & $23.3 \%$ & & & $0.860(0.774-0.955)$ & \\
\hline Stomach, NOS & $26.6 \%$ & & & $0.891(0.801-0.992)$ & \\
\hline Pathological grading & & 98.930 & $<0.001$ & & $<0.001$ \\
\hline High/ Moderate & $37.9 \%$ & & & Reference & \\
\hline Poor/ Anaplastic & $27.4 \%$ & & & $1.156(1.077-1.241)$ & \\
\hline Unknown & $21.7 \%$ & & & $1.186(1.021-1.377)$ & \\
\hline Histotype & & 45.168 & $<0.001$ & & 0.060 \\
\hline Adenocarcinoma & $32.1 \%$ & & & Reference & \\
\hline $\begin{array}{l}\text { Mucinous/signet ring } \\
\text { cell }\end{array}$ & $24.0 \%$ & & & $1.061(0.998-1.129)$ & \\
\hline pT Stage & & 731.610 & $<0.001$ & & $<0.001$ \\
\hline $\mathrm{T} 1$ & $60.2 \%$ & & & Reference & \\
\hline $\mathrm{T} 2$ & $51.1 \%$ & & & $1.156(0.969-1.380)$ & \\
\hline T3 & $31.7 \%$ & & & $1.835(1.584-2.126)$ & \\
\hline $\mathrm{T} 4$ & $17.9 \%$ & & & $2.604(2.246-3.019)$ & \\
\hline $\mathrm{Tx}$ & $4.2 \%$ & & & $4.453(3.216-6.164)$ & \\
\hline pN Stage & & 620.680 & $<0.001$ & & $<0.001$ \\
\hline N1 & $42.9 \%$ & & & Reference & \\
\hline $\mathrm{N} 2$ & $32.2 \%$ & & & $1.371(1.270-1.480)$ & \\
\hline N3a & $19.7 \%$ & & & $2.186(2.010-2.378)$ & \\
\hline $\mathrm{N} 3 \mathrm{~b}$ & $9.2 \%$ & & & $3.524(3.169-2.919)$ & \\
\hline No. of LNs & & 135.822 & $<0.001$ & & $<0.001$ \\
\hline$\leq 6$ & $20.3 \%$ & & & Reference & \\
\hline $7-14$ & $29.0 \%$ & & & $0.586(0.536-0.640)$ & \\
\hline$\geq 15$ & $32.6 \%$ & & & $0.390(0.356-0.427)$ & \\
\hline
\end{tabular}

NI: not included in the multivariate survival analysis.

$P$ values were adjusted for age, race, location, pathological grading, histotype stage, tumor stage and No. of LNs as covariates.

\section{Discussion}

Although the increased trend in the diagnosis of GC, the prognosis of GC is still poor and the 5-year survival was less than 30\% [10]. Radical gastrectomy is considered as the only potentially curative therapy for all the GC patients [11]. LN metastases in gastric cancer are well recognized as one of the most important prognostic factors, and regional lymph nodes dissection could improve the long-term survival $[12,13]$. The American Joint Committee on Cancer (AJCC) has recommended a minimum of 15 
lymph nodes should be examined in order to get accurate postoperative stage $[14,15]$. According to the 8th edition TNM classification, the minimum examined lymph node count is not mandatory for proper staging, although more than 16 examined LNs has been proposed to ensure the accurate prognosis of pN stage since 2009[16]. Moreover, the number of retrieved LNs has been confirmed as an independent prognosis factor in esophageal cancer [17], colon cancer [18] et al. However, debate also exists regarding the importance and the number of retrieved LNs in gastric cancer. Okajima et al. suggested that 25 or more LN harvests might be sufficient for nodal staging [19]. Liu et al. recommended no less than 15 total LNs should be pathologically examined in patients with N1-3 [20]. Shi et al. also reported that negative lymph node counts, which did not take positive LN into consideration, could predict prognosis for patients with gastric cancer [5]. In addition, in node-negative gastric cancer, Zheng et al. found retrieved LN counts was associated with long-time survival outcomes. The higher the LN

A

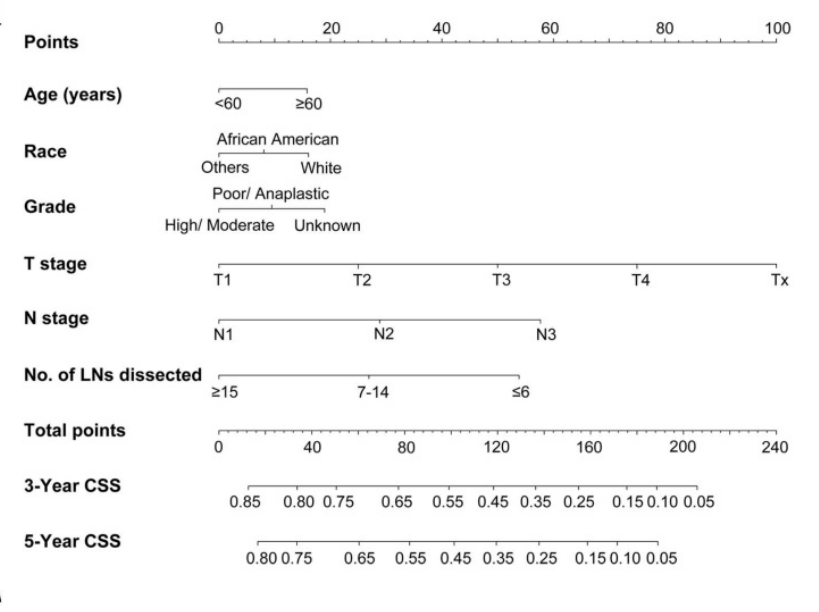

C

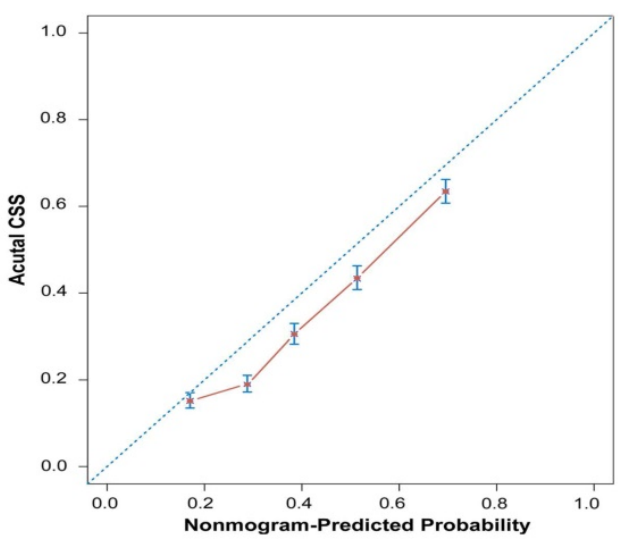

count, the better the survival would be [8]. Deng et al. found that more than 15 examined LNs in node-negative GC patients were mandatory for improvement in the prognostic assessment accuracy [21-23]. However, the relationship between total LN counts and GCSS has not been fully investigated in a large population.

According to all present clinical guidelines, total LN counts for gastric cancer are the main concern. In view of the importance of total LN counts, in this study, we mainly investigate the prognostic value of total LN counts in node-positive GC. We first used the X-tile program to divided GC patients into low, middle, and high-risk groups, and identified 4 and 14 as the optimal cutoff value in terms of GCSS. Then the result was further confirmed in an additional one-by-one cutoff value analysis from 2 to 20. The 5-year GCSS of patients with $\mathrm{N}$ or more nodes increased gradually when $\mathrm{N}$ reached 14 . After the number 15, the survival rates were roughly stable between the compared groups. Above results indicated that inadequate LN harvest in node-positive
B

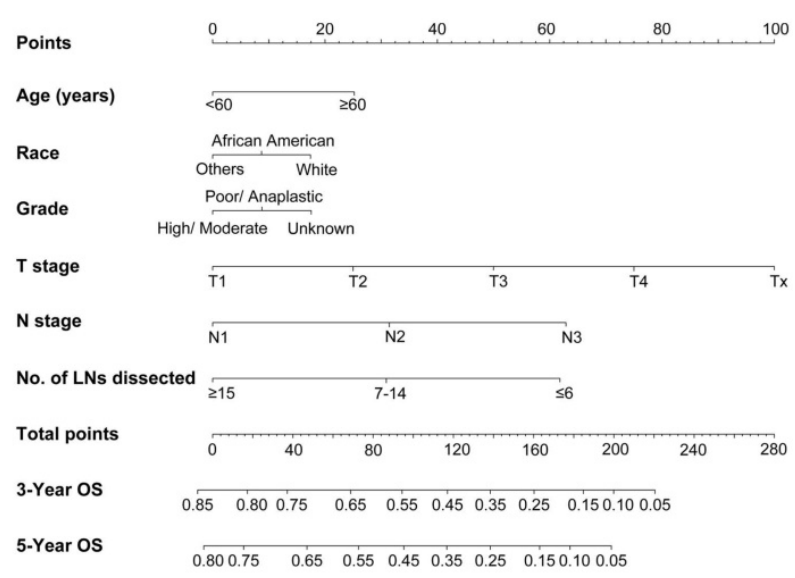

D

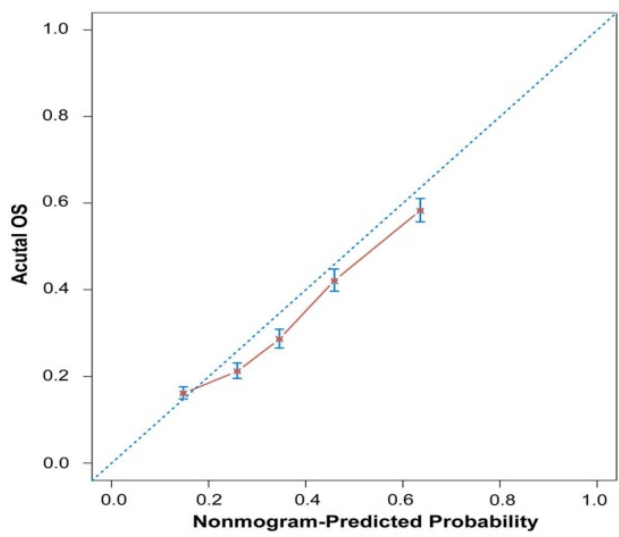

Figure 3. The calibration plots for predicting CSS and OS of gastric cancer patients. Nomograms can be interpreted by summing up the points assigned to each variable, which is indicated at the top of scale. The total points can be converted to predicted 3-year and 5-year probability of survival for a patient in the lowest scale (A, B). The Harrell's c-indexes for CSS and OS prediction were 0.663 ( $95 \% \mathrm{Cl}$ : 0.655-0.671) and 0.654 ( $95 \%$ Cl: 0.646-0.662) (P<0.001), respectively. Calibration curves for 5-year CSS (C) and 5 -year OS (D) using nomograms with clinicopathological characteristics and LN counts are shown. The $x$-axis is nomogram-predicted probability of survival and y-axis is actual survival. The reference line is $45^{\circ}$ and indicates perfect calibration. 
gastric cancer patients may reflect limited lymph node dissection for gastric cancer, which increased the risk of recurrence and metastasis. Besides, we also validated retrieved $\mathrm{LN}$ counts as an independent prognostic factor in node-positive gastric cancer. The survival rates were positively correlated with the number of retrieved LN counts.

Table 4. Univariate and multivariate survival analyses evaluating the number of retrieved LNs influencing GCSS based on different cancer stage.

\begin{tabular}{|c|c|c|c|c|c|}
\hline \multirow[b]{2}{*}{ Variable } & \multirow[b]{2}{*}{ 5-year GCCS } & \multicolumn{2}{|c|}{ Univariate analysis } & \multicolumn{2}{|c|}{ Multivariate analysis } \\
\hline & & Log rank $\chi^{2}$ test & $\mathbf{P}$ & HR(95\%CI) & $\mathbf{P}$ \\
\hline \multicolumn{6}{|l|}{ pN Stage } \\
\hline N1 & & 305.678 & $<0.001$ & & $<0.001$ \\
\hline \multicolumn{6}{|c|}{ No. of LNs } \\
\hline$\leq 6$ & $23.1 \%$ & & & Reference & \\
\hline $7-14$ & $44.3 \%$ & & & $0.581(0.511-0.661)$ & \\
\hline$\geq 15$ & $58.1 \%$ & & & $0.373(0.325-0.427)$ & \\
\hline N2 & & 200.635 & $<0.001$ & & $<0.001$ \\
\hline \multicolumn{6}{|c|}{ No. of LNs } \\
\hline$\leq 6$ & $13.6 \%$ & & & Reference & \\
\hline 7-14 & $29.2 \%$ & & & $0.582(0.507-0.669)$ & \\
\hline$\geq 15$ & $40.7 \%$ & & & $0.406(0.352-0.469)$ & \\
\hline N3 & & 29.113 & $<0.001$ & & $<0.001$ \\
\hline \multicolumn{6}{|c|}{ No. of LNs } \\
\hline \multicolumn{6}{|c|}{$\leq 6$} \\
\hline 7-14 & $10.8 \%$ & & & Reference & \\
\hline$\geq 15$ & $18.3 \%$ & & & $0.789(0.719-0.865)$ & \\
\hline
\end{tabular}

$\mathrm{P}$ values were adjusted for age, race, location, pathological grading, histotype stage and tumor stage as covariates.

NI: not included in the multivariate survival analysis.

The nomogram is a simple statistics-based tool that provides the overall probability of a specific clinical event. For many cancers, nomograms are validated to be more accurate in predicting the probability of an event, such as death or recurrence, when compared with the traditional TNM staging systems [24]. The X-tile software is a comprehensive method, based on traditional statistical tests, and yet intuitive for the oncologist. The X-tile plot illustrates the presence of substantial subpopulations and shows the robustness of the relationship between a biomarker and outcome by construction of a two dimensional projection of every possible sub-population [25]. In this study, we used nomograms incorporating different retrieved LN number that identified the optimal cut-off value by $X$-tile program in a large population, and exhibited better predictive accuracy than that of the model without the variable of dissected LNs.

Several hypotheses may explain this finding for the relationship between the number of retrieved LNs and survival. First, total LN counts indicate the actual harvested LNs number intraoperatively. Moreover, it also reflects the properly identified and examined LNs during pathologic analysis of the surgical specimen, which result in cancer upstaging. Second, previous studies have shown that patients with lymphocytic infiltration have a better survival than those who have no infiltration [26, 27]. More dissected LNs which are associated with LN counts may reflect a higher host lymphocytic reaction to the tumor [28, 29]. Furthermore, we have to remain aware of the fact that increased number of retrieved LNs may attribute to improved surgical techniques. Theoretically, it also reflects an authoritative surgical curability and quality of surgical care or pathology, thus prolonging the survival and disease-free period.

Although this study is based on a large population, there are still potential limitations. First, several important pieces of information regarding surgical options (eg, palliative therapy, radical resection), as well as cancer treatment (chemotherapy, radiotherapy), are not included in the SEER database, which could not be adjusted by our analyses. Second, SEER database also lacks the situation of postoperative adjuvant chemotherapy, and information about the depth of tumor invasion $(\mathrm{T} 4 \mathrm{a} / \mathrm{T} 4 \mathrm{~b})$, as well as the information of pathology-specific covariates including perineural invasion and vascular invasion which are essential for prognosis evaluation. Third, the number of lymph nodes harvested depends on the quality of surgery and pathology. These variables that cannot be adjusted may differ in different institutions. Despite these limitations, our analysis of the SEER database revealed that total $\mathrm{LN}$ counts were an independent prognostic predictor with surgically treated gastric cancer. Increased retrieved LNs count was associated with long-time survival outcomes in node-positive gastric cancer; it could provide more accurate prognostic information than the current node stage system.

\section{Supplementary Material}

Supplementary figure.

http://www.jcancer.org/v10p5646s1.pdf

\section{Acknowledgements}

This work was supported by grants from the National Key Research and Development Program of China (Grant Number: 2016YFC0905900 to B.S.); the State Key Program of National Natural Science Foundation (Grant Number: 81430062 to B.S.); Innovative Research Groups of National Natural Science Foundation (Grant Number: 81521004 to B.S.); The National Natural Science Foundation of China (Grant Number: 81702344 to W.Z.), TianQing Liver Disease Research Fund (Grant Number: TQGB20180095 to W.Z.), B.S. is Yangtze River Scholars Distinguished Professor. 


\section{Author Contributions}

WJZ, GYZY, QXX and BCS designed the study. JCW, GQL, HHC and DCY provided the databases. KPJ, YL, FW, WWY and HTZ assembled and analyzed the data. WJZ, GYZY and QXX wrote the manuscript. All authors read and approved the final manuscript.

\section{Competing Interests}

The authors have declared that no competing interest exists.

\section{References}

1. Jemal A, Bray F, Center MM, Ferlay J, Ward E, Forman D. Global cancer statistics. CA Cancer J Clin. 2011; 61: 69-90.

2. Wroblewski LE, Peek RM, Jr., Wilson KT. Helicobacter pylori and gastric cancer: factors that modulate disease risk. Clin Microbiol Rev. 2010; 23: 713-39.

3. Torres I, Correa P, Ferreccio C, Hernandez-Suarez G, Herrero R, Cavazza-Porro M, et al. Gastric cancer incidence and mortality is associated with altitude in the mountainous regions of Pacific Latin America. Cancer Causes Control. 2013; 24: 249-56.

4. Choi KH, Kim BS, Oh ST, Yook JH. Comparison the sixth and seventh editions of the AJCC staging system for T1 gastric cancer: a long-term follow-up study of 2124 patients. Gastric Cancer. 2016; 20: 43-48.

5. Shi RL, Chen Q, Ding JB, Yang Z, Pan G, Jiang D, et al. Increased number of negative lymph nodes is associated with improved survival outcome in node positive gastric cancer following radical gastrectomy. Oncotarget. 2016; 7: 35084-91.

6. Schwarz RE, Smith DD. Extended lymph node dissection for gastric cancer: who may benefit? Final results of the randomized Dutch gastric cancer group trial. J Clin Oncol. 2005; 23: 5404-5; author reply 5.

7. Smith DD, Schwarz RR, Schwarz RE. Impact of total lymph node count on staging and survival after gastrectomy for gastric cancer: data from a large US-population database. J Clin Oncol. 2005; 23: 7114-24.

8. Zheng WF, Ji TT, Lin Y, Li RZ. The prognostic value of lymph nodes count on survival of patients with node-negative gastric cancer. Oncotarget. 2016; 7: 43680-43688

9. Wang M, Bai J, Tan Y, Wang S, Tian Y, Gong W, et al. Genetic variant in PSCA predicts survival of diffuse-type gastric cancer in a Chinese population. Int J Cancer. 2011; 129: 1207-13.

10. DeSantis CE, Lin CC, Mariotto AB, Siegel RL, Stein KD, Kramer JL, et al. Cancer treatment and survivorship statistics, 2014. CA Cancer J Clin. 2014; 64: 252-71.

11. Jiang L, Yang KH, Guan QL, Zhao P, Chen Y, Tian JH. Survival and recurrence free benefits with different lymphadenectomy for resectable gastric cancer: a meta-analysis. J Surg Oncol. 2013; 107: 807-14.

12. Saito H, Fukumoto Y, Osaki T, Fukuda K, Tatebe S, Tsujitani S, et al. Prognostic significance of level and number of lymph node metastases in patients with gastric cancer. Ann Surg Oncol. 2007; 14: 1688-93.

13. Zhou R, Wu Z, Zhang J, Wang H, Su Y, Huang N, et al. Clinical significance of accurate identification of lymph node status in distant metastatic gastric cancer. Oncotarget. 2016; 7: 1029-41.

14. Washington K. 7th edition of the AJCC cancer staging manual: stomach. Ann Surg Oncol. 2010; 17: 3077-9.

15. Cuschieri SA, Hanna GB. Meta-analysis of D1 versus D2 gastrectomy for gastric adenocarcinoma: let us move on to another era. Ann Surg. 2014; 259: e90.

16. Deng J, Liu J, Wang W, Sun Z, Wang Z, Zhou Z, et al. Validation of clinical significance of examined lymph node count for accurate prognostic evaluation of gastric cancer for the eighth edition of the American Joint Committee on Cancer (AJCC) TNM staging system. Chin J Cancer Res. 2018; 30: 477-91.

17. Hsu PK, Huang CS, Wang BY, Wu YC, Chou TY, Hsu WH. The prognostic value of the number of negative lymph nodes in esophageal cancer patients after transthoracic resection. Ann Thorac Surg. 2013; 96: 995-1001.

18. Lin $X J$, Yu N, Lin $X G$, Zhang $Y F$, Chen $Y$, Zhang $K$, et al. A clinical survey of pain in Parkinson's disease in Eastern China. International psychogeriatrics. 2016; 28 : 283-9.

19. Okajima W, Komatsu S, Ichikawa D, Kosuga T, Kubota T, Okamoto K, et al. Prognostic impact of the number of retrieved lymph nodes in patients with gastric cancer. J Gastroenterol Hepatol. 2016; 31: 1566-71.

20. Liu C, Lu Y, Jun Z, Zhang R, Yao F, Lu P, et al. Impact of total retrieved lymph nodes on staging and survival of patients with gastric cancer invading the subserosa. Surg Oncol. 2009; 18: 379-84

21. Deng J, Yamashita H, Seto Y, Liang H. Increasing the Number of Examined Lymph Nodes is a Prerequisite for Improvement in the Accurate Evaluation of Overall Survival of Node-Negative Gastric Cancer Patients. Ann Surg Oncol. 2017; $24: 745-53$
22. Chu X, Yang ZF. Impact on survival of the number of lymph nodes resected in patients with lymph node-negative gastric cancer. World J Surg Oncol. 2015; 13: 192.

23. Xu D, Huang Y, Geng Q, Guan Y, Li Y, Wang W, et al. Effect of lymph node number on survival of patients with lymph node-negative gastric cancer according to the 7th edition UICC TNM system. PLoS One. 2012; 7: e38681.

24. Sternberg $\mathrm{CN}$. Are nomograms better than currently available stage groupings for bladder cancer? J Clin Oncol. 2006; 24: 3819-20.

25. Camp RL, Dolled-Filhart M, Rimm DL. X-tile: a new bio-informatics tool for biomarker assessment and outcome-based cut-point optimization. Clinical cancer research : an official journal of the American Association for Cancer Research. 2004; 10: 7252-9.

26. Fernandez-Acenero MJ, Galindo-Gallego M, Sanz J, Aljama A. Prognostic influence of tumor-associated eosinophilic infiltrate in colorectal carcinoma. Cancer. 2000; 88: 1544-8.

27. Canna K, McArdle PA, McMillan DC, McNicol AM, Smith GW, McKee RF, et al. The relationship between tumour T-lymphocyte infiltration, the systemic inflammatory response and survival in patients undergoing curative resection for colorectal cancer. Br J Cancer. 2005; 92: 651-4.

28. George S, Primrose J, Talbot R, Smith J, Mullee M, Bailey D, et al. Will Rogers revisited: prospective observational study of survival of 3592 patients with colorectal cancer according to number of nodes examined by pathologists. Br J Cancer. 2006; 95: 841-7.

29. Morris M, Platell C, Iacopetta B. Tumor-infiltrating lymphocytes and perforation in colon cancer predict positive response to 5-fluorouracil chemotherapy. Clin Cancer Res. 2008; 14: 1413-7. 